\title{
The BICEP2 CMB polarization experiment
}

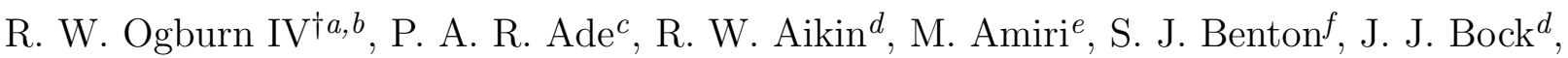
J. A. Bonetti ${ }^{g, d}$, J. A. Brevik ${ }^{d}$, B. Burger ${ }^{e}$, C. D. Dowell ${ }^{d}$, L. Duband ${ }^{h}$, J. P. Filippini ${ }^{d}$,

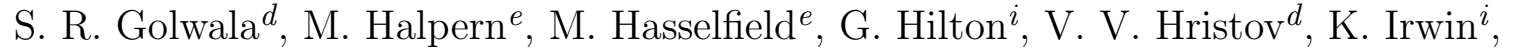
J. P. Kaufman ${ }^{j}$, B. G. Keating ${ }^{j}$. J. M. Kovac ${ }^{k}$, C. L. Kuo ${ }^{a, b}$, A. E. Lange ${ }^{d}$, E. M. Leitch ${ }^{l}$, C. B. Netterfield ${ }^{f}$, H. T. Nguyen ${ }^{g}$, A. Orlando ${ }^{d}$, C. L. Pryke $^{m}$, C. Reintsema ${ }^{h}$, S. Richter ${ }^{k}$, J. E. Ruhl ${ }^{n}$, M. C. Runyan ${ }^{d}$, C. D. Sheehy ${ }^{l, m}$, Z. K. Staniszewski ${ }^{d}$, S. A. Stokes ${ }^{a, b}$, R. V. Sudiwala ${ }^{c}$, G. P. Teply ${ }^{d}$, J. E. Tolan ${ }^{a, b}$, A. D. Turner ${ }^{g}$, P. Wilson ${ }^{g}$, and C. L. Wong ${ }^{k}$

${ }^{a}$ Stanford University, Stanford, 382 Via Pueblo Mall, CA 94305, USA

${ }^{b}$ Kavli Institute for Particle Astrophysics and Cosmology (KIPAC), Sand Hill Road 2575, Menlo Park, CA 94025, USA

${ }^{c}$ Dept. of Physics and Astronomy, Cardiff University, The Parade, Cardiff, CF24 3AA, UK ${ }^{d}$ California Institute of Technology, 1200 E. California Blvd., Pasadena, CA 91125 USA $^{e}$ Department of Physics \& Astronomy, University of British Columbia, 6224 Agricultural Road, Vancouver, BC V6T1Z1, Canada

${ }^{f}$ Department of Physics, University of Toronto, Toronto, ON M5S 1A7, Canada ${ }^{g}$ Jet Propulsion Laboratory, 4800 Oak Grove Dr., Pasadena, CA 91109, USA

${ }^{h}$ Service des Basses Tempratures, DRFMC, CEA-Grenoble, 17 rue des Martyrs, 38054 Grenoble Cedex 9, France

${ }^{i}$ NIST Quantum Devices Group, 325 Broadway, Boulder, CO 80305, USA ${ }^{j}$ University of California, San Diego, La Jolla, CA 92093, USA

${ }^{k}$ Harvard-Smithsonian Center for Astrophysics, 60 Garden Street, Cambridge, MA 02138

${ }^{l}$ Kavli Institute for Cosmological Physics, University of Chicago, 5640 South Ellis Avenue, Chicago, IL 60637, USA

${ }^{m}$ University of Minnesota, Minneapolis, MN 55455, USA

${ }^{n}$ Physics Department, Case Western Reserve University, Cleveland, OH 44106, USA

\begin{abstract}
The BICEP2 telescope is designed to measure the polarization of the cosmic microwave background on angular scales near 2-4 degrees, near the expected peak of the $B$-mode polarization signal induced by primordial gravitational waves from inflation. BICEP2 follows the success of BICEP, which has set the most sensitive current limits on $B$-modes on 2-4 degree scales. The experiment adopts a new detector design in which beam-defining slot antennas are coupled to TES detectors photolithographically patterned in the same silicon wafer, with multiplexing SQUID readout. BICEP2 takes advantage of this design's higher focal-plane packing density, ease of fabrication, and multiplexing readout to field more detectors than BICEP1, improving mapping speed by nearly a factor of 10. BiceP2 was deployed to the South Pole in November 2009 with 500 polarization-sensitive detectors at 150 $\mathrm{GHz}$, and is funded for two seasons of observation. The first months' data demonstrate the performance of the Caltech/JPL antenna-coupled TES arrays, and two years of observation with BICEP2 will achieve unprecedented sensitivity to $B$-modes on degree angular scales.
\end{abstract}

Keywords: Cosmic microwave background, microwave, TES, polarization, inflation, gravitational waves, cosmology

$\dagger^{\dagger}$ Corresponding author: R. W. Ogburn, 382 Via Pueblo Mall, Stanford, CA 94305. E-mail: ogburn@stanford.edu

Millimeter, Submillimeter, and Far-Infrared Detectors and Instrumentation for Astronomy V, edited by Wayne S. Holland, Jonas Zmuidzinas, Proc. of SPIE Vol. 7741, 77411G (c) 2010 SPIE · CCC code: $0277-786$ X/10/\$18 $\cdot$ doi: 10.1117/12.857864

Proc. of SPIE Vol. $774177411 \mathrm{G}-1$ 


\section{INTRODUCTION}

The observed geometric flatness, homogeneity, and isotropy of the Universe can be explained by the idea of cosmological inflation, according to which the observable Universe began as a single microscopic volume and underwent an early period of rapid, exponential expansion. The predictions of the inflationary model for the flatness and uniformity of the Universe, and for the generation of fluctuations, are in good agreement with observations of the cosmic microwave background (CMB). ${ }^{1,2}$ Inflation produces two kinds of fluctuation: scalar density perturbations, which arise from the inflaton field; and tensor metric perturbations, which arise from primordial gravitational waves. The tensor-to-scalar ratio $r$ is governed by the energy scale of the new physics driving inflation. Both tensor and scalar perturbations affect the temperature fluctuations at the time of last scattering, and therefore the temperature and polarization of the CMB. The tensor perturbations induce a unique odd-parity pattern, known as a $B$-mode, on the CMB polarization. If this pattern can be detected, it will provide clear evidence of inflation; either a detection or upper limit can constrain the allowed energy scale of inflation. ${ }^{3}$

The polarization of the CMB was first measured by the DASI experiment, which detected even-parity $E$ modes. ${ }^{4}$ More sensitive instruments are needed to observe the fainter $B$-mode signal; BICEP was designed with this goal in mind. The original BICEP telescope observed from 2006 through 2008 with 49 pairs of polarizationsensitive bolometers (PSBs) fabricated on silicon nitride micromesh and coupled to corrugated feed horns. ${ }^{5,6}$ The results from its first two years of data have set the most sensitive limits on $B$-mode polarization of the CMB in the range $\ell=21-335$, and constrained $r<0.72$ at $95 \%$ confidence level. $^{7}$

BICEP2 builds on the success of BICEP, using the same observing strategy and telescope mount, similar optics, and a similar thermal and cryogenic architecture. BICEP2 observes with 5 times as many detectors, of a new design: Horn-coupled PSBs have been replaced with antenna-coupled TES arrays developed at Caltech and JPL. ${ }^{8}$ BICEP2 uses NIST SQUID amplifiers with the University of British Columbia MCE control and readout electronics. ${ }^{9}$

The experiment was deployed to Amundsen-Scott South Pole Station in November 2009, and commissioned in the Dark Sector Laboratory building in November 2009-January 2010. It observes the CMB in the same primary field as BICEP1, in the Southern Hole, with secondary observations of a bright region of the Galactic plane. We present the experiment and its initial performance characterization in this paper and several companion papers in this volume: the detector design and characterization (Orlando et al. ${ }^{10}$ ), the optical performance (Aikin et $\left.a l .{ }^{11}\right)$, and noise levels and sensitivity (Brevik et al. ${ }^{12}$ ).

\section{INSTRUMENT DESIGN}

The results of BICEP1 have demonstrated the power of its experimental approach, and in particular the ability to achieve very high sensitivity while controlling systematics. BICEP2 is designed for similar sensitivity and low systematic contaminations, with increased number of pixels and hence mapping speed. The entire focal plane observes in a single frequency band $(150 \mathrm{GHz})$ in a patch of sky with very low dust and synchrotron emission, expected to have Galactic foreground confusion below $r \sim 0.03$ (see Fig. 1). This experiment opens a path to larger arrays with greater sensitivity: The Keck Array and SpIDER telescopes will use the same detector design, but with multiple focal planes, including some at 100 and $220 \mathrm{GHz}$ to allow foreground removal. ${ }^{13}$

\subsection{Detectors}

The higher detector count of BICEP2 is made possible by combining the functions of several components, which required separate construction and hand-assembly in BICEP1, into a single silicon wafer. The beam-defining feed horns are replaced with a network of slot antennas; the micromesh absorber, with a resistive gold meander; and the NTD bolometer, with a Ti transition-edge sensor. These components are all photolithographically patterned in a single fabrication process. The technology of antenna-coupled TES detectors with multiplexed readout has allowed BICEP2 to increase the pixel count of BICEP1 by a factor of five. The instrument contains four Si tiles, each with 64 dual-polarization spatial pixels in an $8 \times 8$ array, for a total of 500 optically coupled, $150 \mathrm{GHz}$ detectors and 12 dark TES detectors. A single pixel consists of two orthogonal antenna networks, each coupled through a feed line and a lumped-element band-defining filter to a Ti transition-edge sensor (TES) suspended on a SiN membrane. The sensors have $T_{c}$ of $500-524 \mathrm{mK}$ and $G_{c} \approx 100 \mathrm{pW} / \mathrm{K}$. The dark pixels are identical, 


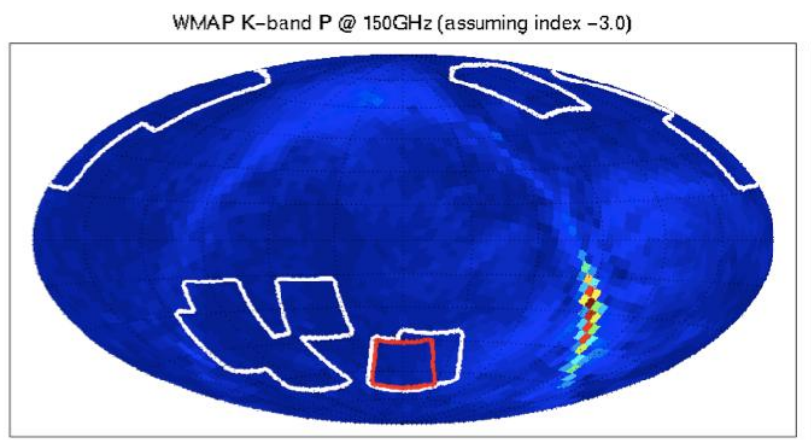

Color range 0 to $4 \mu \mathrm{K}$

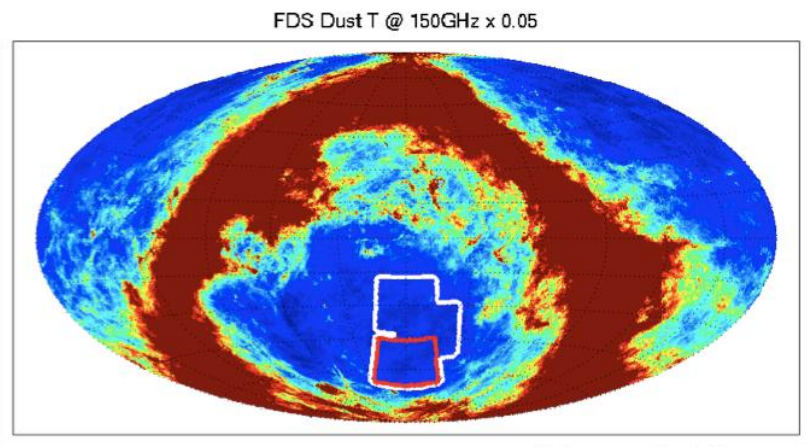

Color range 0 to $4 \mu \mathrm{K}$

Figure 1. The primary BICEP CMB field (outline in red) has very low foreground contamination from synchrotron emission (left) and dust (right). The synchrotron signal is estimated from the WMAP K-band maps, extrapolated to $150 \mathrm{GHz}$ with an assumed spectral index of -3 . The dust signal is the FDS model value for $150 \mathrm{GHz}$ with an assumed $5 \%$ polarization fraction. The $150 \mathrm{GHz}$ band is near the expected minimum for the sum of dust and synchrotron foregrounds.

except that the band-defining filter is replaced with an electrical break. Two of the detector tiles have one dark pixel each, and the other two tiles have two dark pixels each.

The detector tiles were extensively characterized at Caltech and JPL during 2008-2009, and because the Caltech/JPL detector technology allows rapid fabrication, several improvements and adjustments were incorporated into the final set of detectors deployed in BICEP2. A separate paper in this volume by A. Orlando details the design of the antenna-coupled TES arrays, along with detector characterization and design modifications from the commissioning of BICEP2. ${ }^{10}$

\subsection{Optics}

The optical design is similar to BICEP1: we use on-axis, refractive optics located inside the dewar. Resolving the target 2-4 degree-scale CMB modes does not require large optics; we use lenses and an aperture of $30 \mathrm{~cm}$ diameter for beams with $\sigma \approx 0.2^{\circ}$. The advantages of this relatively simple optical design are that it is efficient to assemble and transport, all optics can be cooled to $4 \mathrm{~K}$ for stability and low loading, and the beams can be measured in the far field $(>50 \mathrm{~m})$ using controlled sources on the ground.

The telescope contains two lenses, the eyepiece and objective lens, made from high-density polyethylene. The optical system is telecentric in the image space. In order to reduce loading on the focal plane, we use Teflon filters. Pre-deployment testing showed that the detectors have a small out-of-band coupling just above the high-frequency cutoff, with cross-polarization leakage. To attenuate this signal, we have added a metal mesh low-pass edge filter manufactured by P. Ade at Cardiff University, ${ }^{14}$ with a cutoff at $8.3 \mathrm{~cm}^{-1}$. The vacuum window is $32 \mathrm{~cm}$ in diameter and $12 \mathrm{~cm}$ thick, made of laminated Zotefoam PPA30.* The window is sealed to its Al housing with Stycast 1266 epoxy.

A full description of the BICEP2 optics is given in Aikin et al. in these proceedings. ${ }^{11}$

\subsection{Cryogenics and thermal design}

The telescope is housed within a liquid helium cryostat from Redstone Aerospace ${ }^{\dagger}$, very similar to the BICEP1 dewar. The major change is that the liquid nitrogen stage of BICEP1 has been replace with a vapor-cooled shield, so that LHe is the only consumable cryogen. The helium reservoir has a capacity of $100 \mathrm{~L}$, and consumes about $22 \mathrm{~L} /$ day during ordinary observing.

The focal plane is cooled by a Duband three-stage ${ }^{4} \mathrm{He} /{ }^{3} \mathrm{He} /{ }^{3} \mathrm{He}$ sorption refrigerator that operates in a closed cycle. Several improvements have been made in the thermal path between the refrigerator and the focal

\footnotetext{
*Zotefoams Inc., Walton, KY 41094. Phone: 800-362-8358. Web: http://zotefoams.com/

${ }^{\dagger}$ http://www.redstoneaerospace.com/
} 
plane relative to BICEP, giving BICEP2 improved stability and reduced microphonic pickup. The coldest stage of the fridge is linked to the focal plane through a passive thermal filter in the form of a stainless steel block, $5.5 \mathrm{~cm}$ in length and a $2.5 \mathrm{~cm} \times 2.5 \mathrm{~cm}$ square in cross-section. Achieving large enough thermal conduction with a long time constant requires the filter to have high heat capacity and low thermal diffusivity; it can develop a significant thermal gradient across its length. While some materials (such as holmium) can have lower thermal diffusivity, 316 stainless steel was chosen as a readily available material with suitable thermal properties for this application. The design approach of the passive filter was inspired by the distributed thermal filter used in the Planck HFI instrument. ${ }^{15,16}$ The filter has a complex transfer function, and effectively isolates the focal plane from thermal fluctuations faster than about $1300 \mathrm{~s}$.

The thermal straps have been redesigned as flexible stacks of many layers of high-conductivity copper foil, which reduce the microphonic sensitivity relative to the stiffer linkages used in BICEP. The focal plane is supported using a carbon fiber truss structure, which provides good thermal isolation from the $4 \mathrm{~K}$ stage. ${ }^{17}$

With no additional heating, the focal plane achieves a base temperature of $\sim 250 \mathrm{mK}$. In observing mode, resistive heaters on the focal plane and on the fridge side of the thermal filter are continuously servoed to keep the copper plate of the focal plane at a constant $280 \mathrm{mK}$, well below the $500-\mathrm{mK}$ Ti transition temperature. Any residual sensitivity to thermal fluctuations or changes in loading can be characterized by NTD thermometers mounted on each detector tile, and dark sensors, which are identical to the polarization-sensitive pixels in every way, except that the microstrip connection to the antennas is broken.

The refrigerator can be recycled in slightly under 4 hours, and subsequently remains at a stable base temperature for at least 80 hours. The recycling operation, helium fill, and occasional star observations (to verify pointing stability) are performed every three sidereal days, during a block of six hours set aside for maintenance.

\subsection{Multiplexing readout}

The antenna-coupled TES detectors measure a time-varying microwave signal as a modulation in the current passing through the sensor. This is amplified within the cryostat using a 3-stage SQUID multiplexer from NIST. ${ }^{18}$ Each TES is coupled by an input inductor to a first-stage SQUID, and each column of 33 first-stage SQUIDs is fed into a summing coil and second-stage SQUID. The first- and second-stage SQUIDs are on the focal plane, at $280 \mathrm{mK}$. The signals are amplified in a series SQUID array at $4 \mathrm{~K}$ before readout by room-temperature electronics. This NIST time-domain multiplexing system has gone through many generations of development, and understanding of its performance including expected levels of crosstalk is mature. ${ }^{19}$

For the biasing and multiplexed readout of the TES detectors and SQUID system, we have adopted the University of British Columbia multi-channel electronics (MCE) system. ${ }^{9}$ The MCE hardware is contained in a $6 \mathrm{U}$ crate attached to the bottom of the cryostat, which communicates with the control and data acquisition computers via three optical fibers.

Table 1. Multiplexing parameters used by BICEP2

\begin{tabular}{|l|l|}
\hline Raw ADC sample rate & $50 \mathrm{MHz}$ \\
\hline Row dwell time & 98 samples $\times 20 \mathrm{~ns}$ \\
\hline Row switching rate & $510 \mathrm{kHz}$ \\
\hline Number of rows & 33 \\
\hline Row revisit rate & $15.46 \mathrm{kHz}$ \\
\hline Internal downsample & 150 \\
\hline Output data rate per channel & $103 \mathrm{~Hz}$ \\
\hline Software downsample & 5 \\
\hline Archived data rate & $20.6 \mathrm{~Hz}$ \\
\hline
\end{tabular}




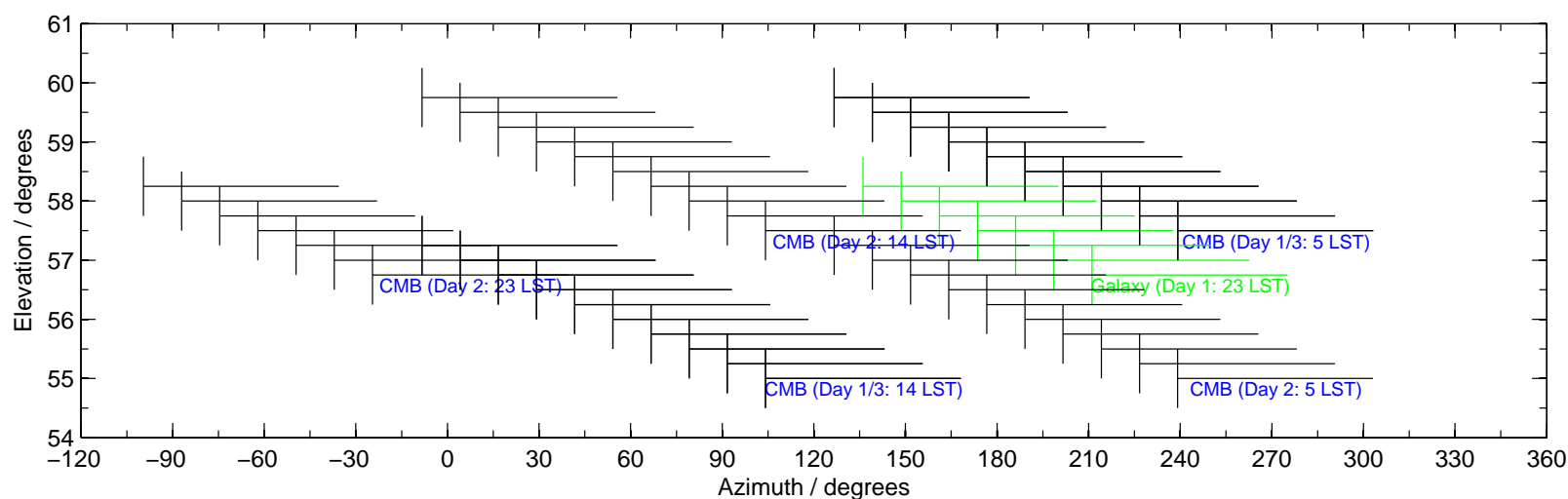

Figure 2. Scanning pattern of a 72-hr observing schedule. The cycle consists of a 6-hr cryogenic maintenance period (not shown) and two 9-hr blocks of raster scans on the CMB on the first day; a 6-hr block of similar raster scans on the Galaxy and two more 9-hr blocks on the main CMB field on the second day; and a 6-hr block and two 9-hr blocks of scans on the CMB on the third day. Each horizontal line is a 50 -minute raster or scan set, with a $\pm 0.6^{\circ}$ elevation "nod" at the beginning and end to measure the relative gains between pixels and between the $\mathrm{Q}$ and $\mathrm{U}$ channels within a polarization pair. The $72-\mathrm{hr}$ schedule is repeated at four boresight rotation angles: $68^{\circ}, 113^{\circ}, 248^{\circ}$, and $293^{\circ}$.

The MCE controls the SQUID multiplexer stages and reads out the signal from a $33 \times 16$ array of firststage SQUIDs through 16 readout channels. The multiplexing parameters in BICEP2 configuration are shown in Table 1. A four-pole Butterworth filter with $f_{3 \mathrm{~dB}}=38.7 \mathrm{~Hz}$ is applied internally before downsampling to $100 \mathrm{~Hz}$. Most of these parameters can be configured live; the raw rate is fixed, and large changes to the internal MCE downsample require a straightforward firmware change.

\subsection{Magnetic sensitivity}

Each of the first-, second-, and third-stage SQUIDs, as well as each summing coil, is a sensitive flux-to-voltage transducer, susceptible to pickup of ambient magnetic fields including that of the Earth. This appears as an additive signal that is (for a given channel) a fixed function of horizontal coordinates. We minimize the effect of magnetic pickup at three levels: first, in the manufacture of the SQUIDs themselves; second, by surrounding the receiver with passive magnetic shielding; and third, by removing the magnetic signal during data reduction.

The SQUIDs made at NIST are gradiometric, rejecting uniform fields and first-order gradients. Each SQUID chip contains 33 first-stage SQUIDs for 32 TES detectors plus one dark channel, for which the input inductor is left electrically open. The dark SQUIDs are multiplexed and read out as usual, through the same secondand third-stage SQUIDs as the bonded channels. Any magnetic signal will appear similar in the light and dark channels, but thermal and optical signals are absent in the dark SQUIDs. This allows characterization and (if needed) subtraction of any residual magnetic pickup.

The internal magnetic shielding includes superconducting and high-permeability components. The multiplexing chips on the focal plane are backed with sheets of Metglas 2714A, which were found to reduce pickup at the first-stage SQUIDs more effectively than niobium foil. A Nb backshort sits behind the focal plane, and a cylindrical $\mathrm{Nb}$ shield surrounds it. The cylindrical shield includes a plate with a square window, which opens immediately in front of the detector tiles. The entire 4-K telescope assembly is shielded with an open-ended cylinder of Cryoperm 10 high-permeability alloy. ${ }^{\ddagger}$ The series SQUID arrays at the $4 \mathrm{~K}$ stage are shielded with superconducting niobium sleeves and wrapped in Metglas 2714A, which is found to to attenuate magnetic sensitivity to a level much lower than that at the first and second stages.

The residual magnetic sensitivity is still significant, as large as $\Delta T^{\mathrm{CMB}}=4 \mathrm{mK}$ across the width of a scan. This is consistent with pre-deployment testing, which indicates that the pickup is primarily at the second-stage SQUID or the summing coil. These are common to all detectors in the same multiplexing column, so that any magnetic pickup is common-mode among each group of 33 channels.

\footnotetext{
${ }^{\ddagger}$ Amuneal Manufacturing Corp., 4737 Darrah Street, Philadelphia, PA 19124, USA, http://amuneal.com/
} 
The remaining signal must be removed in analysis. There are four steps in the standard BICEP data processing which naturally remove magnetic pickup signals: scan-fixed signal subtraction, pair differencing, and polynomial filtering. In addition, the dark SQUID signals can be used to subtract the magnetic signal from the time streams. Scan-fixed signal subtraction takes advantage of the BICEP observing pattern, in which each 50-minute scan set rasters across a fixed range of azimuth at a constant elevation. Any signal caused by external magnetic fields (as well as ground pickup or scan-related thermal fluctuations) will naturally be synchronous relative to the scan and relative to the ground within the scan set. Since the magnetic signal remains fixed in horizontal coordinates, while the sky drifts past, it is accounted for and removed by the same template-subtraction scheme used by Bicep1. Pair differencing applies to the $Q$ and $U$ maps, which are made from pair-differenced time streams. Since the magnetic signal is common-mode within each multiplexing column, this pair-differencing automatically removes it from the polarized signal. Polynomial filtering is applied in mapmaking, and removes long-period trends from the scans. The Earth's field enters as a simple sinusoid, well outside the science band, and will be removed. If necessary, the signals from the dark SQUIDs can be used to create a new magnetic template, which can be subtracted from the optically coupled pixels.

\subsection{Telescope mount}

The telescope mount from BICEP1 remains installed in the Dark Sector Laboratory, and the BiCEP2 dewar inserted into it in place. The major modification is the replacement of a slip ring, used in BICEP1 to allow unlimited rotation about the boresight, with a cylindrical drum through which the readout and control cables are fed. This accommodates the much larger bundle of cables needed for the BICEP2 housekeeping system, while retaining a range of rotation of $380^{\circ}$. Our selection of boresight angles for observing therefore remains unrestricted.

\section{OBSERVING STRATEGY}

BICEP2 uses an expanded version of the BICEP1 observing pattern, with improved efficiency because cryogenic servicing is needed only every three days instead of every two days. During each period of three sidereal days we fully cover the main CMB field three times (54 hours); perform one additional six-hour partial observation of the CMB field; observe the galactic plane for another six-hour period; and stop at zenith for six hours to allow for the liquid helium fill, refrigerator recycling, and star pointing.

\subsection{Observing fields}

The primary science field is the same as in BICEP1: a region of 800 degree $^{2}$ centered at $\left(\mathrm{RA}=0 \mathrm{hr}, \operatorname{dec}=-57.5^{\circ}\right)$. This field, which falls within the "Southern Hole," has been chosen to minimize possible foregrounds to the $B$ mode signal. Gravitational lensing can mix $E$-modes into $B$-modes, contributing more strongly at higher $\ell^{3}$ Our CMB field is large enough to study modes below $\ell=100$, where the lensing background is smaller relative to primordial $B$-modes. ${ }^{20}$ Galactic dust is more than a factor of 100 lower in the Southern Hole than the sky median, as shown in the right pane of Fig. $1 .^{21}$ If the dust emission is assumed to be polarized at $5 \%$, the contribution of dust at $150 \mathrm{GHz}$ is at $r<0.02$. The synchrotron contribution is not well measured, but can be constrained to a level similar to the dust at $150 \mathrm{GHz}{ }^{13}$

In addition to the $\mathrm{CMB}$ region, we also observe a bright section of the galactic plane in a secondary field, centered at $\left(\mathrm{RA}=15: 42 \mathrm{hr}\right.$, dec $\left.=-55.0^{\circ}\right)$, for six hours out of every three days.

\subsection{Scan calibration}

The BICEP scan pattern is built around periods of scanning at a fixed elevation, within a fixed range in azimuth. The scan rate is $2.8^{\circ} / \mathrm{s}$ in azimuth, which corresponds to a modulation at $5 \mathrm{~Hz}$ for $\ell=300$. This sets the upper limit of the necessary science bandwidth. The turn-around portion of the scan has been optimized during BICEP2 commissioning. The resulting improvement in total observing efficiency is $4 \%$. BICEP2 now spends $22.3 \%$ of each scan in the unused end portion. This figure is weakly analysis-dependent, since the data reduction pipeline may apply a tighter or looser cut around the scan turn-arounds.

A 50-minute raster pattern of 53 scans, with endpoints and center fixed in horizontal coordinates, composes one scan set. Each scan set is preceded and followed by bracketing calibrations: an elevation nod of $1.2^{\circ}$, and 


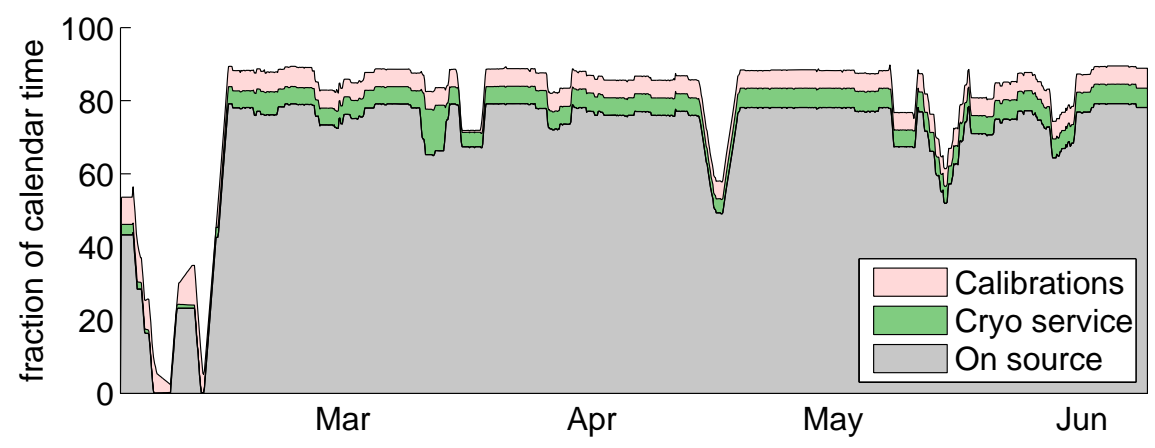

Figure 3. Time spent in CMB scans, regular calibrations, and cryogenic servicing since February 1, 2010. During January and the first half of February, observing schedules were interspersed with beam mapping and other tests and calibrations. Since February 15, the on-source efficiency has been high. The remaining time not categorized in this figure includes scheduled pauses for detector biasing, thermal settling, and telescope slewing; the part of the 6-hour cryogenic block not used by the fridge recycle, which ordinarily requires 4 hours; and time spent on other calibration and testing tasks.

an $I$ - $V$ sweep of each TES device. These allow us to track any drift in detector loading, responsivity, and bias point.

After the trailing calibrations, the telescope steps up by $0.25^{\circ}$ degree in elevation, the scan center is adjusted to match the new center of the observing field, and another 50 -minute scan set is taken. This scanning pattern ensures that any ground pickup, magnetic signal, or scan-synchronous thermal or microphonic effect remain fixed in azimuth during each 50-minute scan set, while the CMB drifts past. These sources of contaminant signal can then be effectively fitted and subtracted out, as was demonstrated in BICEP.

A block of 10 scan sets composes a 9-hour phase, and a block of 7 scan sets makes up a 6-hour phase. A single 9-hour CMB observing phase covers either the top half or the bottom half of the primary field, and a 6 -hour phase can cover the bright Galactic secondary field, or add additional integration on the central part of the CMB field.

A 9-hour observing phase contains 10 scan sets, and a 6 -hour phase contains 7 sets, along with accompanying calibrations. A full observing cycle spans three sidereal days and eight phases. During this time BICEP2 performs six 9-hour phases in the CMB field, covering the field fully three times; an additional 6-hour phase partly covering the CMB field; a six-hour phase in the bright galactic field; and a 6-hour maintenance period. During a given three-day schedule, the boresight rotation is fixed at one of the four angles $68^{\circ}, 113^{\circ}, 248^{\circ}$, or $293^{\circ}$.

The increase in cryogenic hold time allows us to spend up to $79.2 \%$ of calendar time scanning on-source. In practice, this is reduced by time spent in instrument characterization and other interruptions to the observing schedule. As of early June 2010, BICEP2 has accumulated 1841 hours of scanning on-source in the primary CMB field, and 284 hours in the bright galactic region. The efficiency has been good after the initial commissioning period: $74.6 \%$ of calendar time since Feb. 15 has been spent on-source, including the CMB and galactic fields, as shown in Fig. 3. When the turn-around efficiency is taken into account, the ideal total observing efficiency is $61 \%$.

\subsection{Calibration}

Gain calibrations follow directly from the experience of BICEP. Absolute calibration of the detector gains comes from the cross-correlation of BICEP2 CMB maps with those from the WMAP satellite, ${ }^{22}$ as performed for the BICEP results. ${ }^{7}$ Relative calibrations are integrated into the observing schedule. Each 50 -minute scan set is preceded by an "up-down-up" elevation nod, a one-minute atmospheric response test in which the telescope slews upward by $0.6^{\circ}$, downward by $1.2^{\circ}$, and back upward by $0.6^{\circ}$ to return to the observing point. The scan set is followed by a reverse, "down-up-down" elevation nod. As in BICEP, the bracketing elevation nods serve several purposes: 1) to determine the relative gains of the two polarization channels within a pixel, to allow 
pair differencing; 2) to determine the relative gains between pixels, to allow them to be weighted correctly when making maps; and 3) to reject data in which responsivity is anomalous or unstable.

Along with the elevation nods, each scanset is bracketed with partial load curves of the TES detectors. These are performed by increasing the TES bias voltage to drive the detectors normal, and ramping the voltage down until it reaches the operating bias. This provides an $I-V$ characteristic for each sensor, and an effective measurement of the optical loading.

Every 6- or 9-hour observing phase begins with an extended version of these calibrations. The elevation nod is replaced with a "sky dip", in which the telescope slews from an elevation of $50^{\circ}$ up to zenith and back. This provides a profile of the atmospheric conditions. The load curve does not stop at the operating bias, but instead, the detector bias is ramped down to zero, so that each TES enters the superconducting state. This gives a full $I-V$ characteristic including the entire transition region, at the expense of the additional time required to re-bias the detectors afterward.

The polarization efficiencies of the detectors have been measured using a linearly polarized source mounted to a mast above the Martin A. Pomerantz Observatory (MAPO). The cross-polar response has been constrained to $\leq 5 \times 10^{-3} \cdot 11$

\section{PERFORMANCE}

\subsection{Optics and beams}

The optical performance of the telescope has been extensively characterized by near-field and far-field beam mapping, both at Caltech and after deployment at the South Pole. These tests have revealed two significant deviations from ideal beams shapes and centers, which could contribute systematic errors in the CMB polarization measurement. The first is a "beam-steering" in the near field, in which the beams are displaced from the center of the aperture. This causes increased ellipticity, and may cause far-field differences between the beam shapes for the two polarizations of a pixel. This effect may be caused by a gradient in index of refraction of a dielectric underlying the antennas.

The second effect is a pointing offset between the beams in the far field. This differential pointing can be as large as $10 \%$ of the beam width. The mismatch is nearly constant in amplitude and direction across the detectors in the focal plane. This holds even though two of the detector tiles have their orientation rotated by $180^{\circ}$ relative to the other two. Since the offset does not rotate along with the tiles, the effect appears to arise from one or more components of the optical stack.

These effects and approaches for mitigating them, along with ideas for correcting them for the second season of BiceP2, are covered more fully in the Aikin et al. paper on optical design and performance. ${ }^{11}$

From Fourier-transform spectroscopy, the bands are centered at $148 \mathrm{GHz}$.

\subsection{Noise and sensitivity}

The paper by Brevik in these proceedings presents the detector noise level and initial estimates of mapping speed. ${ }^{12}$ The power spectrum of noise in the detector timestreams has been modeled and is consistent with expectations, and a map-based comparison shows that BICEP2 improves on the mapping speed of BICEP1 by a factor of 8-10. This statistical estimate does not account for systematic effects, which are still being characterized.

\section{INITIAL MAPS}

The initial data from BICEP2 have been given a preliminary analysis with blind pointing and no deconvolution of detector time constants. The relative gains are derived from elevation nod responses, and the deglitching and filtering are as in the BICEP1 analysis.

Fig. 4 shows the bright galactic field as imaged after 58 hours of integration. The polarization of the Galactic emission is caused by spinning dust grains aligned in the magnetic field of the Milky Way.

A preliminary CMB temperature map is shown in Fig. 5. The fluctuations are a good match to the CMB field as imaged by BICEP1 and to the data from WMAP when re-observed with the BICEP2 scan strategy. 


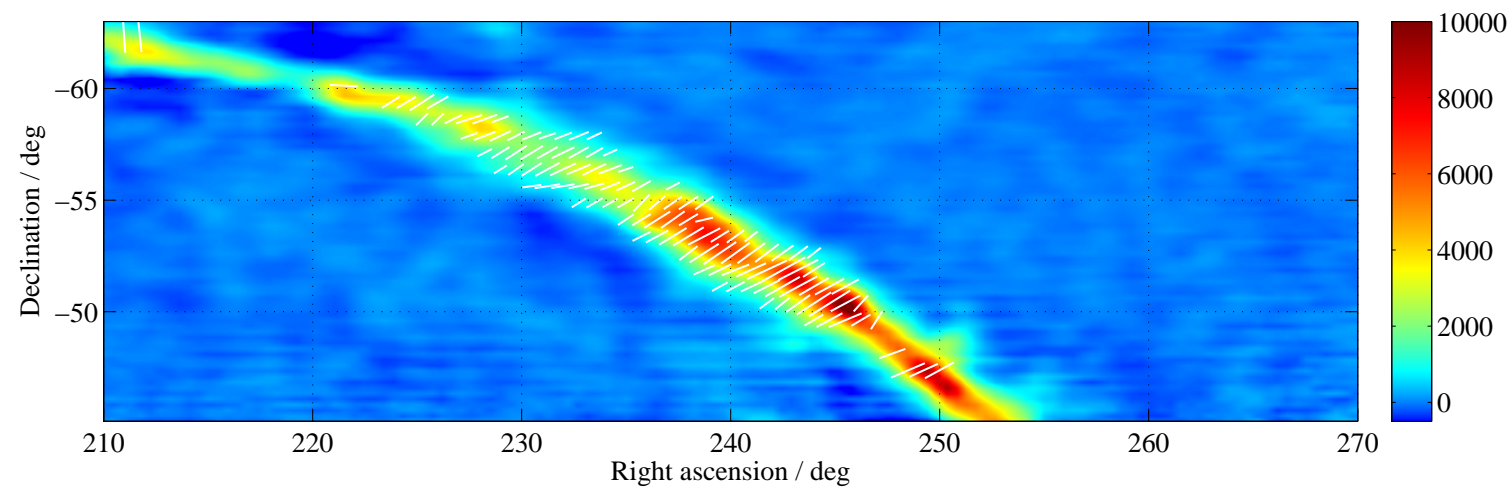

Figure 4. This "first light" map of the bright Galactic field was made with data from February 2010. Microwave radiation emitted by spinning dust grains, aligned in the magnetic field of the galaxy, is about 1-3\% polarized; white line segments show the direction and magnitude of polarization.

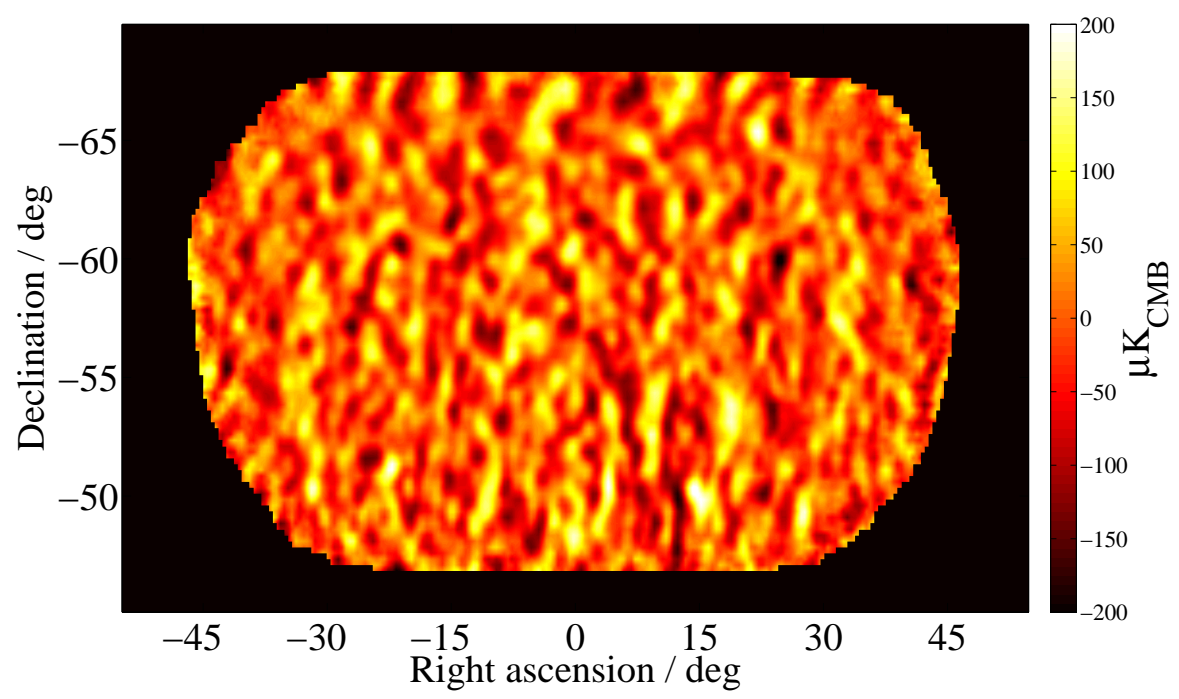

Figure 5. Preliminary results from BICEP2 observations of the primary CMB field. This temperature map is made with 1564 hours of integration on source, from February 1 through June 15. 


\section{CONCLUSION}

BICEP2 was deployed to the South Pole in November 2009 as an upgrade to the successful BiCEP CMB polarization experiment, and has been observing since February 2010. The instrument is performing well, and has collected over 1800 hours of integration on the primary CMB field. Preliminary estimates show a factor of 8-10 improvement in mapping speed relative to BICEP. Far-field beam maps show a consistent offset of the A and B beams, the systematic contributions of which are currently being characterized. BICEP2 is currently funded to observe through the 2010 and 2011 seasons, with a possible upgrade during summer of 2010-11.

\section{ACKNOWLEDGMENTS}

This paper is dedicated to the memory of Andrew Lange, who died under tragic circumstances in January 2010. Andrew made invaluable contributions to the field of experimental cosmology. He played a central role in the conception of the BICEP2 and Keck experiments. His scientific aptitude, leadership, and unique abilities to recognize and develop young scientists are sorely missed by his friends and colleagues.

BICEP2 has been made possible by support from the National Science Foundation, Grant No. ANT-0742818. Detector development has been made possible by the generous support of the Gordon and Betty Moore Foundation. RWO gratefully acknowledges support from the Kavli Institute for Particle Astrophysics and Cosmology. We are grateful to have Steffen Richter as our 2010 South Pole winter-over. The BICEP2 team would also like to thank the South Pole Station staff for logistical support. We thank our BICEP, Keck Array, and SPIDER col-

leagues for useful discussions and shared expertise. Finally we wish to thank Barbara Wertz and Kathy Deniston for their tireless logistical and administrative support.

\section{REFERENCES}

[1] de Bernardis, P., Ade, P. A. R., Bock, J. J., Bond, J. R., Borrill, J., Boscaleri, A., Coble, K., Crill, B., Gasperis, G. D., Farese, P., Ferreira, P., Ganga, K., Giacometti, M., and Hivon, E., "A flat universe from high-resolution maps of the cosmic microwave background radiation," Nature 404, 69-81 (2000).

[2] Peiris, H. V., Komatsu, E., Verde, L., Spergel, D. N., Bennett, C. L., Halpern, M., Hinshaw, G., Jarosik, N., Kogut, A., Limon, M., Meyer, S. S., Page, L., Tucker, G. S., Wollack, E., and Wright, E. L., "First-year Wilkinson Microwave Anisotropy Probe (WMAP) Observations: Implications for inflation," The Astrophysical Journal Supplement Series 148(1), 213 (2003).

[3] Hu, W. and White, M., "A CMB polarization primer," New Astronomy 2, 323-344 (1997).

[4] Kovac, J. M. et al., "Detection of polarization in the cosmic microwave background using DASI," Nature 420, $722-787$ (2002).

[5] Yoon, K. W., Ade, P. A. R., Barkats, D., Battle, J. O., Bierman, E. M., Bock, J. J., Brevik, J. A., Chiang, H. C., Crites, A., Dowell, C. D., Duband, L., Griffin, G. S., Hivon, E. F., Holzapfel, W. L., Hristov, V. V., Keating, B. G., Kovac, J. M., Kuo, C. L., Lange, A. E., Leitch, E. M., Mason, P. V., Nguyen, H. T., Ponthieu, N., Takahashi, Y. D., Renbarger, T., Weintraub, L. C., and Woolsey, D., "The Robinson Gravitational Wave Background Telescope (BICEP): a bolometric large angular scale CMB polarimeter," in [Society of Photo-Optical Instrumentation Engineers (SPIE) Conference Series], Presented at the Society of Photo-Optical Instrumentation Engineers (SPIE) Conference 6275, 62751K (July 2006).

[6] Takahashi, Y. D. et al., "Characterization of the BICEP telescope for high-precision cosmic microwave background polarimetry," Astrophys. J 711, 1141-1156 (2010).

[7] Chiang, H. C. et al., "Measurement of cosmic microwave background polarization power spectra from two years of BICEP data," Astrophys. J 711, 1123-1140 (2010).

[8] Orlando, A. et al., "Antenna-coupled TES arrays for the BICEP2/Keck and SPIDER polarimeters," AIP Conference Proceedings 1185, 471-474 (2009).

[9] Battistelli, E. S. et al., "Functional description of read-out electronics for time-domain multiplexed bolometers for millimeter and sub-millimeter astronomy," J. Low Temp. Phys. 151, 908-914 (2008).

[10] Orlando, A. et al., "Antenna-coupled TES bolometer arrays for BICEP2/Keck and SPIDER," These proceedings (2010). 
[11] Aikin, R. W. et al., "Optical performance of the BICEP2 telescope at the South Pole," These proceedings (2010).

[12] Brevik, J. A. et al., "Initial performance of the BICEP2 antenna-coupled superconducting bolometers at the South Pole," These proceedings (2010).

[13] Nguyen, H. T., Kovac, J., Ade, P., Aikin, R., Benton, S., Bock, J., Brevik, J., Carlstrom, J., Dowell, D., Duband, L., Golwala, S., Halpern, M., Hasslefield, M., Irwin, K., Jones, W., Kaufman, J., Keating, B., Kuo, C.-L., Lange, A., Matsumura, T., Netterfield, B., Pryke, C., Ruhl, J., Sheehy, C., and Sudiwala, R., "BICEP2/SPUD: searching for inflation with degree scale polarimetry from the South Pole," Millimeter and Submillimeter Detectors and Instrumentation for Astronomy IV 7020(1), 70201F, SPIE (2008).

[14] Ade, P. A. R., Pisano, G., Tucker, C., and Weaver, S., "A review of metal mesh filters," in [Society of Photo-Optical Instrumentation Engineers (SPIE) Conference Series], Presented at the Society of PhotoOptical Instrumentation Engineers (SPIE) Conference 6275 (July 2006).

[15] Piat, M., Lamarre, J.-M., Meissonnier, J., Torre, J.-P., Camus, P., Benoit, A., Crussaire, J.-P., Ade, P. A. R., Bock, J. J., Lange, A. E., Bhatia, R., Maffei, B., Puget, J. L., and Sudiwala, R. V., "Planck-HFI thermal architecture: from requirements to solutions," IR Space Telescopes and Instruments 4850(1), 740-748, SPIE (2003).

[16] Heurtel, A. and Piat, M., "0.1 K cooler / $\mathrm{Ho}_{x} \mathrm{Y}_{1-x}$ FPU struts: Product assurance specifications," Planck HFI internal note (Apr. 2000).

[17] Runyan, M. and Jones, W., "Thermal conductivity of thermally-isolating polymeric and composite structural support materials between 0.3 and $4 \mathrm{~K}, "$ Cryogenics 48(9-10), 448 - 454 (2008).

[18] Irwin, K. D., Vale, L. R., Bergren, N. E., Deiker, S., Grossman, E. N., Hilton, G. C., Nam, S. W., Reintsema, C. D., Rudman, D. A., and Huber, M. E., "Time-division SQUID multiplexers," Low Temperature Detectors 605, 301-304 (Feb. 2002).

[19] de Korte, P. A. J., Beyer, J., Deiker, S., Hilton, G. C., Irwin, K. D., Macintosh, M., Nam, S. W., Reintsema, C. D., Vale, L. R., and Huber, M. E., "Time-division superconducting quantum interference device multiplexer for transition-edge sensors," Review of Scientific Instruments 74, 3807-3815 (Aug. 2003).

[20] Smith, K. M., Hu, W., and Kaplinghat, M., "Weak lensing of the CMB: Sampling errors on B modes," Phys. Rev. D 70, 043002 (Aug 2004).

[21] Finkbeiner, D. P., Davis, M., and Schlegel, D. J., "Extrapolation of Galactic Dust Emission at 100 Microns to CMBR Frequencies Using FIRAS," Astrophys. J. 524, 867-886 (1999).

[22] Jarosik, N., Bennett, C. L., Dunkley, J., Gold, B., Greason, M. R., Halpern, M., Hill, R. S., Hinshaw, G., Kogut, A., Komatsu, E., Larson, D., Limon, M., Meyer, S. S., Nolta, M. R., Odegard, N., Page, L., Smith, K. M., Spergel, D. N., Tucker, G. S., Weiland, J. L., Wollack, E., and Wright, E. L., "Seven-Year Wilkinson Microwave Anisotropy Probe (WMAP) Observations: Sky Maps, Systematic Errors, and Basic Results," ArXiv e-prints (Jan. 2010). 